\title{
PD-1 inhibitor combined with apatinib for advanced gastric or esophagogastric junction cancer: a retrospective study
}

\author{
Qing Wei ${ }^{1,2 *}$, Xing Yuan ${ }^{1,2 *}$, Jingjing $\mathrm{Li}^{1,2}$, Qi Xu${ }^{1,2}$, Jieer Ying ${ }^{1,2}$ \\ ${ }^{1}$ Department of Abdominal Medical Oncology, Cancer Hospital of the University of Chinese Academy of Sciences (Zhejiang Cancer Hospital), \\ Hangzhou, China; ${ }^{2}$ Institute of Cancer and Basic Medicine (ICBM), Chinese Academy of Sciences, Hangzhou, China \\ Contributions: (I) Conception and design: Q Wei, X Yuan, J Ying; (II)Administrative support: J Li, Q Xu; (III) Provision of study materials or patients: \\ All authors; (IV) Collection and assembly of data: All authors; (V) Data analysis and interpretation: Q Wei, X Yuan; (VI) Manuscript writing: All \\ authors; (VII) Final approval of manuscript: All authors. \\ "These authors contributed equally to this work. \\ Correspondence to: Jieer Ying; Qi Xu. Department of Abdominal Medical Oncology, Cancer Hospital of the University of Chinese Academy of \\ Sciences (Zhejiang Cancer Hospital), Hangzhou, China; Institute of Cancer and Basic Medicine (ICBM), Chinese Academy of Sciences, Hangzhou, \\ China. Email: jieerying@aliyun.com; hzxuqi@sina.cn.
}

\begin{abstract}
Background: Nivolumab and pembrolizumab were approved as immune checkpoint inhibitors for thirdline treatment of advanced gastric or esophagogastric junction cancer (GC/EGJC) in 2017. However, immunotherapy monotherapy has low efficacy. Apatinib has been proven effective in advanced GC/EGJC. Numerous studies have shown that immunotherapy has a synergistic effect when combined with targeted drug therapy. Based on these facts and to assess the efficacy and safety of programmed death 1 (PD1) inhibitor and apatinib as combination therapy in patients (pts) with unresectable locally advanced or metastatic GC/EGJC, a retrospective clinical research study was carried out.
\end{abstract}

Methods: Pts ( $\mathrm{n}=24)$ received PD-1 inhibitor and apatinib (250 mg once daily) as second- or third-line therapy in this observational, retrospective study. The primary objectives were efficacy and safety.

Results: At data cut-off (December 31, 2019), 24 pts were enrolled. Of the 19 pts who were evaluable, the objective response rate (ORR) was 26.3\% (5/19), the median progression-free survival (PFS) was $3.0(95 \%$ CI: 1.3 to 4.7 ) months, and the median overall survival (OS) was not reached. Grade 3 or 4 treatment-related adverse events (TRAEs) occurred in 3 (15.8\%) of the 19 pts. These adverse events (AEs) included pruritus, rash, hand-foot syndrome, and increased aspartate aminotransferase (AST) or alanine aminotransferase (ALT). No treatment-related deaths occurred.

Conclusions: Combination therapy of PD-1 inhibitor and apatinib showed encouraging clinical activity and demonstrated tolerable toxicity in pts with advanced GC/EGJC. Hence, our work provide rationale for the combination of PD-1 inhibitor and apatinib in advanced GC/EGJC.

Keywords: Programmed death 1 (PD-1); gastric cancer (GC); immune checkpoint inhibitors; immunotherapy; apatinib

Submitted Mar 04, 2020. Accepted for publication Jul 14, 2020.

doi: $10.21037 /$ tcr-20-1333

View this article at: http://dx.doi.org/10.21037/tcr-20-1333

\section{Introduction}

Gastric or esophagogastric junction cancer (GC/EGJC) is the third leading cause of cancer-related deaths worldwide, with most of the cases diagnosed at late stages $(1,2)$. For patients (pts) with unresectable or recurrent advanced
GC/EGJC, systemic chemotherapy is the most important method used to prolong survival (3). However, research data indicate that the objective response rate (ORR) ranges from $6.8-25 \%$ and the progression-free survival (PFS) was $1.5-5.3$ months in second or further lines therapy (4-6), 
showing a poor prognosis. With this background, new clinical treatment approaches for advanced GC/EGJC are urgently needed, particularly in later lines. Immune checkpoint inhibitors have been shown to be effective in treating GC through blocking the interaction between programmed cell death-1 (PD-1) and its ligand (PDL1) $(7,8)$. Of these, nivolumab and pembrolizumab, were approved as third-line therapies for advanced GC/EGJC in 2017. However, only about $10 \%$ of advanced GC/ EGJC patients benefit from monotherapy overall $(9,10)$. Therefore, to extend the benefit to a larger population, the development of innovative strategies such as combining $\mathrm{PD}-1 / \mathrm{PD}-\mathrm{L} 1$ blockade with conventional treatments is urgently needed in advanced GC/EGJC.

In recent years, studies have shown that immunotherapy has a synergistic effect when combined with molecular antiangiogenic agents $(11,12)$. Anti-angiogenesis is a well-established tumor microenvironment (TME) targeted therapy in GC/EGJC. Combining PD-1/PD-L1 blockade with agents that can eliminate the preexisting immunosuppression of TME may overcome the primary resistance in patients with advanced GC/EGJC (13-15). Moreover, in preliminary results from an open-label clinical trial, with a combination of anti-PD-1 antibody and VEGFR1-3 inhibitor, nivolumab plus regorafenib achieved an ORR of 44\% (5/9) in pts with pretreated GC (16), which provides rationale to apply immunotherapy combined with molecular antiangiogenic agents for GC/EGJC.

Apatinib, a small-molecule anti-angiogenesis targeted drug, has been approved as third-line or above therapy for pts with advanced GC/EGJC in China (17). In in vitro studies, apatinib and $\mathrm{PD}-1$ inhibitor have shown complementary anti-tumor effects $(18,19)$. Based on these results, we carried out a retrospective clinical research study to assess the value of clinical application of PD-1 inhibitor and apatinib as combination therapy in pts with advanced GC/EGJC. We present the following article in accordance with the STROBE reporting checklist (available at http:// dx.doi.org/10.21037/tcr-20-1333).

\section{Methods}

\section{Study population}

Our study collected 24 pts with histologically confirmed, unresectable locally advanced or metastatic HER2-negative GC/EGJC treated with PD-1 inhibitor combined with apatinib in Zhejiang Cancer Hospital from May 2018 to
May 2019. The study was conducted in accordance with the Declaration of Helsinki (as revised in 2013). The study was approved by the Ethics Committee of Zhejiang Cancer Hospital (IRB-2019-155) and written informed consent was obtained from all patients. The Eastern Cooperative Oncology Group (ECOG) performance status of 0-2 was selected. Other inclusion criteria included PD-1 inhibitor combined with apatinib for more than two cycles, and at least one target lesion that could be measured by imaging. Pts with autoimmune diseases and who had received any PD-1, PD-L1, or other drug immunotherapy were excluded. Further details are shown in Table 1.

\section{Study design and assessments}

Our study is a retrospective, single-center study. Pts received apatinib at doses of $250 \mathrm{mg}$ in combination with PD-1 inhibitor including SHR-1210 (200 mg Q2W), nivolumab (3 mg/kg Q2W), JS001 (240 mg Q3W), or sintilimab (200 mg Q3W). Treatment was continued until disease progression, intolerable toxicity, or other reason for termination was judged by the investigator.

The efficacy and safety of PD-1 inhibitor plus apatinib in advanced GC/EGJC pts are the primary objectives to be evaluated. Tumor assessments were performed through CT or MRI after every two cycles of treatment according to the RECIST v1.1 guideline. Observed indicators included ORRs, disease control rates (DCRs), PFS, and overall survival (OS). Adverse events (AEs) were assessed according to the Common Terminology Criteria for Adverse Events (version 4.0). If any grade $\geq 3 \mathrm{AEs}$ occurred, apatinib or PD-1 inhibitor were discontinued until the adverse reaction returned to $\leq 1$ degree. If the adverse reactions caused by apatinib lead to treatment delays of more than 4 weeks, apatinib was discontinued.

\section{Statistical analysis}

The distributions of PFS and OS were estimated using the Kaplan-Meier (KM) method. The statistical significance of survival curves was tested with a log-rank test. All data were analyzed by SPSS 20 statistical analysis.

\section{Results}

\section{Patient information and baseline characteristics}

Twenty-four pts were enrolled to receive SHR-1210 ( $\mathrm{n}=2)$, 
Table 1 Patient information and baseline characteristics

\begin{tabular}{|c|c|}
\hline Characteristic & No $(\%)(n=24)$ \\
\hline Age, years, median [range] & $60.5[30-74]$ \\
\hline \multicolumn{2}{|l|}{ Gender } \\
\hline Male & $18(75.0)$ \\
\hline Female & $6(25.0)$ \\
\hline \multicolumn{2}{|l|}{ ECOG } \\
\hline 0 & $4(16.7)$ \\
\hline 1 & $16(66.6)$ \\
\hline 2 & $4(16.7)$ \\
\hline \multicolumn{2}{|c|}{ Histology subtype (Lauren classification) } \\
\hline Intestinal & $5(20.8)$ \\
\hline Diffuse & $10(41.7)$ \\
\hline Mixed & $4(16.7)$ \\
\hline Unknown & $5(20.8)$ \\
\hline \multicolumn{2}{|l|}{ Number of metastatic sites } \\
\hline $1-2$ & $9(37.5)$ \\
\hline$\geq 3$ & $15(62.5)$ \\
\hline \multicolumn{2}{|l|}{ Peritoneal metastases } \\
\hline Yes & $17(70.8)$ \\
\hline No & $7(29.2)$ \\
\hline \multicolumn{2}{|l|}{ Liver metastases } \\
\hline Yes & $13(54.2)$ \\
\hline No & $11(45.8)$ \\
\hline \multicolumn{2}{|l|}{ Prior therapies } \\
\hline Surgery & $14(58.3)$ \\
\hline 1st line therapy & $16(66.6)$ \\
\hline$>1$ st line therapy & $8(33.4)$ \\
\hline \multicolumn{2}{|l|}{ Immunotherapy drugs } \\
\hline JS001 & $15(62.5)$ \\
\hline Sintilimab & $6(25.0)$ \\
\hline Nivolumab & $1(4.2)$ \\
\hline SHR-1210 & $2(8.3)$ \\
\hline
\end{tabular}

ECOG, Eastern Cooperative Oncology Group.

nivolumab ( $\mathrm{n}=1)$, JS001 $(\mathrm{n}=15)$, or sintilimab $(\mathrm{n}=6)$, until the data cutoff (December 31, 2019). The median age was 60.5 (range, 30-74) years and 75\% (18/24) were male.

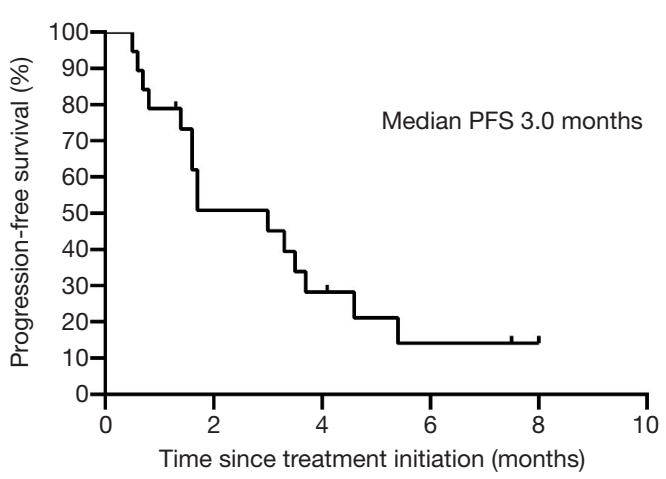

Figure 1 KM plot of PFS. KM, Kaplan-Meier; PFS, progressionfree survival.

Fifteen $(62.5 \%)$ pts had multiple metastatic lesions $(\geq 3)$ and $58.3 \%(14 / 24)$ pts had undergone surgery. Of the 24 pts, 16 $(66.6 \%)$ and $8(33.4 \%)$ pts had previously received first-line treatment and more than first-line treatment, respectively. Additional details are provided in Table 1.

\section{Efficacy}

Of 24 pts, 19 pts were evaluable by RECIST v1.1. One patient achieved complete response (CR), four pts achieved partial response (PR), seven pts achieved stable disease (SD), and seven pts had progressive disease (PD). The ORR was $26.3 \%(5 / 19)$, and the DCR was $63.2 \%(12 / 19)$.

Median time to response was 1.7 (interquartile range, 1.6 to 2.1) months. Median duration of response was 3.0 (interquartile range, 1.8 to 3.7 ) months. The median PFS was 3.0 (95\% CI, 1.3 to 4.7 ) months (Figure 1, Table 2). The median OS was not reached (Table 2).

Specifically to explain the efficacy and changes in tumor regression, we show the best percentage change in lesion size of the 19 pts in our study in Figure $2 A$ and percentage changes over time in Figure $2 B$.

\section{Safety}

Safety analysis of this study in 19 pts is as follows. Treatment-related adverse events (TRAEs) leading to discontinuation were reported in 3 (15.8\%) of the 19 pts. These included liver function damage [increased aspartate aminotransferase (AST) and alanine aminotransferase (ALT)], rash and pruritus, and hand-foot syndrome. Allgrade TRAEs reported in $\geq 5 \%$ of patients are summarized in Table 3. Relatively few grade 3 or 4 TRAEs occurred, 
Table 2 Efficacy of PD-1 and apatinib combination treatment in pts with GC/EGJC

\begin{tabular}{|c|c|}
\hline Evaluation & Value \\
\hline \multicolumn{2}{|c|}{ RECIST v1.1 tumor evaluation } \\
\hline CR & 1 \\
\hline PR & 4 \\
\hline $\mathrm{SD} \geq 6$ weeks & 7 \\
\hline PD & 7 \\
\hline Not evaluable & 5 \\
\hline ORR in evaluable patients & $26.30 \%$ \\
\hline DCR in evaluable patients & $63.20 \%$ \\
\hline Median time to response & 1.7 months \\
\hline \multicolumn{2}{|l|}{ Duration of response } \\
\hline $\mathrm{KM}$ median & 3.0 months \\
\hline \multicolumn{2}{|l|}{ PFS } \\
\hline KM median & 3.0 months \\
\hline \multicolumn{2}{|l|}{ OS } \\
\hline KM median & NR \\
\hline \multicolumn{2}{|c|}{$\begin{array}{l}\text { GC, gastric cancer; EGJC, esophagogastric junction cancer; } \\
\text { CR, complete response; PR, partial response; SD, stable } \\
\text { disease; PD, progressive disease; ORR, overall response rate; } \\
\text { DCR, disease control rate; KM, Kaplan-Meier; PFS, progression- } \\
\text { free survival; OS, overall survival; NR, not reached. }\end{array}$} \\
\hline
\end{tabular}

but these included pruritus (5.3\%), rash (5.3\%), handfoot syndrome (5.3\%), and increased AST (5.3\%) and ALT $(5.3 \%)$. No treatment-related deaths occurred.

\section{Discussion}

In this retrospective study, the ORR following PD-1 inhibitor and apatinib combination therapy was $26.3 \%$, and the PFS was 3.0 months. Compared with previous study results, in which that ORR ranges from $6.8-25 \%$ and the PFS was 1.5-5.3 months (4-6), a significant increase of ORR was shown. Simultaneously, DCR was observed in $63.2 \%(12 / 19)$ of pts in our study, with median duration of response of 3.0 (interquartile range, 1.8 to 3.7 ) months. Long-lasting responses existed. In a study of apatinib monotherapy for advanced GC/EGJC in third-line and above treatment, the median PFS was 2.6 months, ORR was $2.84 \%$, and DCR was $42.05 \%$ (20). Our results show that the efficacy of PD-1 inhibitor and apatinib combination therapy turned out to be better compared to apatinib monotherapy.

Nivolumab and pembrolizumab have been approved as immune checkpoint inhibitors for third-line treatment indications for advanced GC/EGJC. But till now, the ORR of immunotherapy monotherapy is only $11-23 \%$ (21-23), which emphasizes the necessity of changing treatment options to improve efficacy. Results of this research show that PD-1 inhibitor and apatinib combination therapy improve the efficacy of treatment, mainly because first, tumor angiogenesis inhibits the extravasation of reactive $\mathrm{T}$ cells, which form an immunosuppressive microenvironment that leads to tumors escaping immunosurveillance. Combination therapy strengthens T-cell infiltration and activation to eliminate tumor cells (24-27). In addition, Jain et al. and Huang et al. demonstrated that anti-angiogenic therapy causes vascular normalization, mitigating hypoxia, and may allow more effective $\mathrm{T}$ cells to extravasate from the blood into the TME and enhance cancer immunotherapies $(28,29)$. Moreover, anti-vascular targeted therapy apatinib may enhance anti-tumor immune responses by breaking oncogene dependence, which, in turn, causes cancer cell senescence and promotes T-cell clearance (30). Zhao and his team found that low-dose apatinib $(250 \mathrm{mg} / \mathrm{d})$ could impede the recruitment of tumor-associated macrophages, decrease TGF- $\beta$ level, block tumor growth and metastasis, and eventually cause prolonged survival in mouse and in vitro models (31).

The safety profile of combination therapy in pts with advanced GC/EGJC was manageable. Adverse reactions are controllable. The types of adverse reactions were consistent with those known to be related to PD-1 inhibitor and apatinib $(7,9,20,23)$. Thyroid disorders are associated with PD-1 inhibitor. All of the adverse effects we found were grades 1 and 2 . This may be related to the possibility that PD-1 inhibitors may modulate the immune balance and stimulate their own immune potential (32). We found that hand-foot syndrome, proteinuria, decreased platelet count, leukopenia, and neutropenia were associated with apatinib. TRAEs leading to discontinuation were reported in three pts. These three pts had to discontinue treatment due to liver function damage (AST and ALT increases), rash and pruritus, and hand-foot syndrome. It seems that combination therapy leads to a slight increase in adverse reactions, including ALT and AST increases. No treatmentrelated deaths occurred. The combination therapy is safe and reliable in clinical application.

There were some limitations in our research. First, 

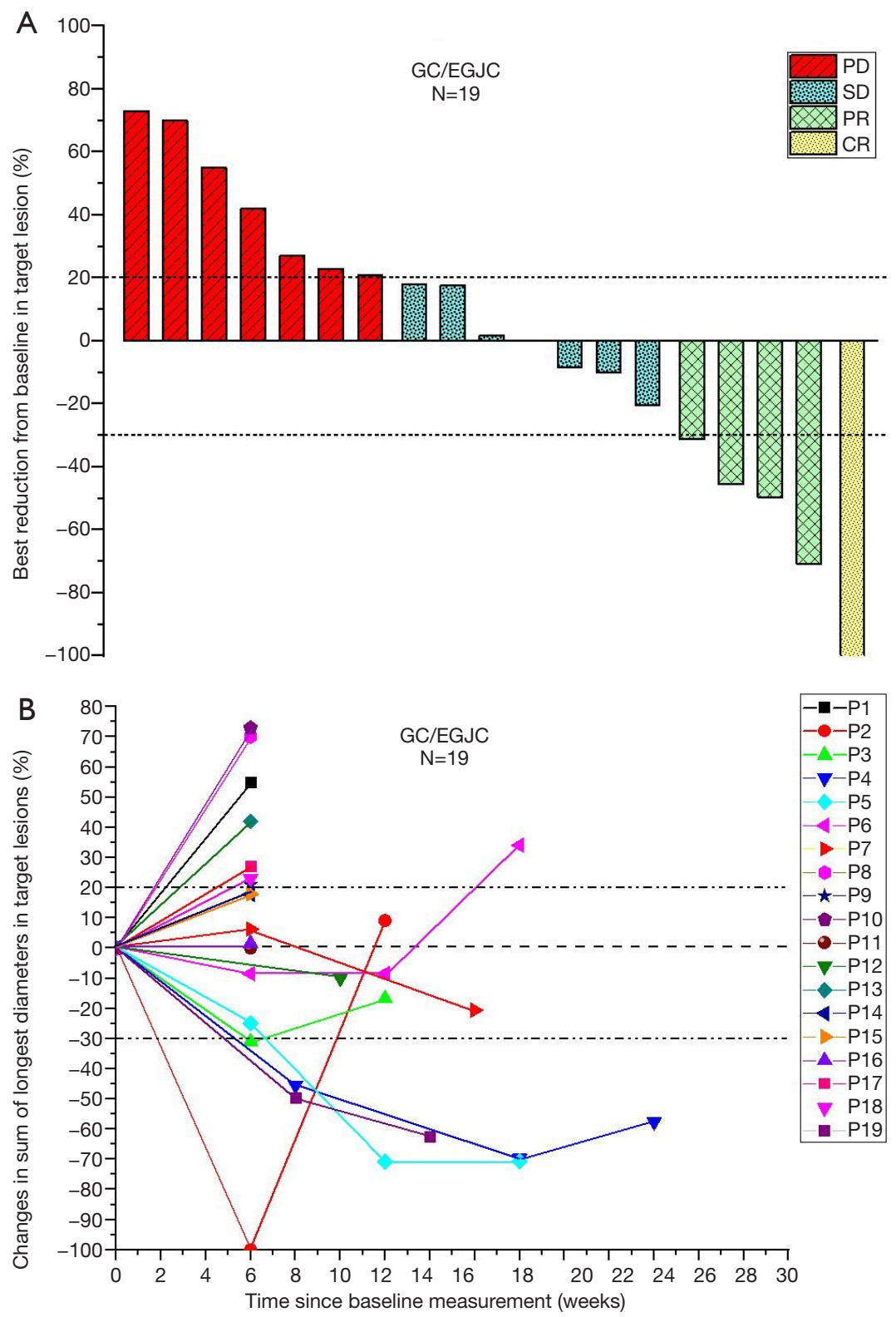

Figure 2 Best percentage change in size of target lesion and lesion diameters over time. (A) Waterfall plot of best percentage change from baseline in size of target lesion; (B) percentage change of lesion diameters over time. GC, gastric cancer; EGJC, esophagogastric junction cancer.

this report was a single-center retrospective study with insufficient sample and possibly incomplete information, resulting in recall bias. Additionally, we lacked data, including biomarkers PD-L1 and tumor mutation burden, which would have been related to the efficacy of immunological checkpoint inhibitors. We will explore this further in the future.
In conclusion, PD-1 inhibitor and apatinib combination therapy has shown encouraging clinical activity, can improve survival, and demonstrates tolerable toxicity in pts with advanced GC/EGJC as second- or third-line therapy. We expect further research, especially in the field of firstline or neoadjuvant therapy, to continue exploring the value of this combination therapy in advanced GC/ EGJC. 
Table 3 Treatment-related adverse events (TRAEs)

\begin{tabular}{|c|c|c|}
\hline & \multicolumn{2}{|c|}{ Total $(n=19), n(\%)$} \\
\hline & Any grade & Grade 3/4 \\
\hline TRAEs & $3(15.8)$ & $3(15.8)$ \\
\hline \multicolumn{3}{|l|}{ Common TRAE } \\
\hline Decreased appetite & $5(26.3)$ & 0 \\
\hline Diarrhea & $3(15.8)$ & 0 \\
\hline Nausea & $3(15.8)$ & 0 \\
\hline Fatigue & $6(31.6)$ & 0 \\
\hline Vomiting & 4 (21.1) & 0 \\
\hline Abdominal pain & $3(15.8)$ & 0 \\
\hline Pyrexia & $1(5.3)$ & 0 \\
\hline Pruritus & $2(10.5)$ & $1(5.3)$ \\
\hline Rash & $2(10.5)$ & $1(5.3)$ \\
\hline Hand-foot syndrome & 4 (21.1) & $1(5.3)$ \\
\hline Proteinuria & $1(5.3)$ & 0 \\
\hline AST increase & $7(36.8)$ & $1(5.3)$ \\
\hline Blood bilirubin increase & $6(31.6)$ & 0 \\
\hline ALT increase & $7(36.8)$ & $1(5.3)$ \\
\hline \multicolumn{3}{|l|}{ Hematological AE } \\
\hline Platelet count decrease & $5(26.3)$ & 0 \\
\hline Leukopenia decrease & $4(21.1)$ & 0 \\
\hline Neutropenia decrease & $4(21.1)$ & 0 \\
\hline Hemoglobin decrease & $3(15.8)$ & 0 \\
\hline \multicolumn{3}{|c|}{ Additional TRAEs of special interest } \\
\hline Interstitial lung disease & 0 & 0 \\
\hline Colitis & 0 & 0 \\
\hline Hypopituitarism & 0 & 0 \\
\hline Thyroid disorder & $8(42.1)$ & 0 \\
\hline
\end{tabular}

AE, adverse event; ALT, alanine aminotransferase; AST, aspartate aminotransferase.

\section{Acknowledgments}

We thank all researchers and patients for participating in this trial.

Funding: This work was supported by Zhejiang Chinese Medicine Science and Technology Program (grant number 2018ZB022).

\section{Footnote}

Reporting Checklist: The authors have completed the STROBE reporting checklist. Available at http://dx.doi. org/10.21037/tcr-20-1333

Data Sharing Statement: Available at http://dx.doi. org/10.21037/tcr-20-1333

Conflicts of Interest: All authors have completed the ICMJE uniform disclosure form (available at http://dx.doi. org/10.21037/tcr-20-1333). The authors have no conflicts of interest to declare.

Etbical Statement: The authors are accountable for all aspects of the work in ensuring that questions related to the accuracy or integrity of any part of the work are appropriately investigated and resolved. The study was conducted in accordance with the Declaration of Helsinki (as revised in 2013). The study was approved by the Ethics Committee of Zhejiang Cancer Hospital (IRB-2019155 ) and written informed consent was obtained from all patients.

Open Access Statement: This is an Open Access article distributed in accordance with the Creative Commons Attribution-NonCommercial-NoDerivs 4.0 International License (CC BY-NC-ND 4.0), which permits the noncommercial replication and distribution of the article with the strict proviso that no changes or edits are made and the original work is properly cited (including links to both the formal publication through the relevant DOI and the license). See: https://creativecommons.org/licenses/by-nc-nd/4.0/.

\section{References}

1. Ferlay J, Soerjomataram I, Dikshit R, et al. Cancer incidence and mortality worldwide: sources, methods and major patterns in GLOBOCAN 2012. Int J Cancer 2015;136:E359-86.

2. Catalano V, Labianca R, Beretta GD, et al. Gastric cancer. Crit Rev Oncol Hematol 2009;71:127-64.

3. Niccolai E, Taddei A, Prisco D. Gastric cancer and the epoch of immunotherapy approaches. World J Gastroenterol 2015;21:5778-93.

4. Chan WL, Yuen KK, Siu SW, et al. Third-line systemic treatment versus best supportive care for advanced/ 
metastatic gastric cancer: a systematic review and metaanalysis. Crit Rev Oncol Hematol 2017;116:68-81.

5. Galdy S, Cella CA, Spada F, et al. Systemic therapy beyond first-line in advanced gastric cancer: an overview of the main randomized clinical trials. Crit Rev Oncol Hematol 2016;99:1-12.

6. Takahari D. Second-line chemotherapy for patients with advanced gastric cancer. Gastric Cancer 2017;20:395-406.

7. Magalhães H, Fontes-Sousa M, Machado $M$. Immunotherapy in advanced gastric cancer: an overview of the emerging strategies. Can J Gastroenterol Hepatol 2018;2018:2732408.

8. Matsueda S, Graham DY. Immunotherapy in gastric cancer. World J Gastroenterol 2014;20:1657-66.

9. Kang YK, Boku N, Satoh T, et al. Nivolumab in patients with advanced gastric or gastro-oesophageal junction cancer refractory to, or intolerant of, at least two previous chemotherapy regimens (ONO-4538-12, ATTRACTION-2): a randomised, double-blind, placebocontrolled, phase 3 trial. Lancet 2017;390:2461-71.

10. Fashoyin-Aje L, Donoghue M, Chen H et al. FDA approval summary: pembrolizumab for recurrent locally advanced or metastatic gastric or gastroesophageal junction adenocarcinoma expressing PD-L1. Oncologist 2019;24:103-9.

11. Manegold C, Dingemans AC, Gray JE, et al. The potential of combined immunotherapy and antiangiogenesis for the synergistic treatment of advanced NSCLC.J Thorac Oncol 2017;12:194-207.

12. Zitvogel L, Galluzzi L, Smyth MJ, et al. Mechanism of action of conventional and targeted anticancer therapies: reinstating immunosurveillance. Immunity 2013;39:74-88.

13. Vanneman M, Dranoff G. Combining immunotherapy and targeted therapies in cancer treatment. Nat Rev Cancer 2012;12:237-51.

14. Hamzah J, Jugold M, Kiessling F, et al. Vascular normalization in Rgs5-deficient tumours promotes immune destruction. Nature 2008;453:410-4.

15. Tartour E, Pere H, Maillere B, et al. Angiogenesis and immunity: a bidirectional link potentially relevant for the monitoring of antiangiogenic therapy and the development of novel therapeutic combination with immunotherapy. Cancer Metastasis Rev 2011;30:83-95.

16. Fukuoka S, Hara H, Takahashi N, et al. Regorafenib plus nivolumab in patients with advanced gastric or colorectal cancer: an open-label, dose-escalation, and dose-expansion phase Ib trial (REGONIVO, EPOC1603). J Clin Oncol 2020;38:2053-61.
17. Roviello G, Ravelli A, Polom K, et al. Apatinib: a novel receptor tyrosine kinase inhibitor for the treatment of gastric cancer. Cancer Letters 2016;372:187-91.

18. Schoenfeld JD, Dranoff G. Anti-angiogenesis immunotherapy. Hum Vaccin 2011;7:976-81.

19. Yasuda S, Sho M, Yamato I, et al. Simultaneous blockade of programmed death 1 and vascular endothelial growth factor receptor 2 (VEGFR2) induces synergistic antitumour effect in vivo. Clin Exp Immunol 2013;172:500-6.

20. Li J, Qin S, Xu J, et al. Randomized, double-blind, placebo-controlled phase III trial of apatinib in patients with chemotherapy-refractory advanced or metastatic adenocarcinoma of the stomach or gastroesophageal junction. J Clin Oncol 2016;34:1448-54.

21. Coutzac C, Pernot S, Chaput N. Immunotherapy in advanced gastric cancer, is it the future? Crit Rev Oncol Hematol 2019;133:25-32.

22. Fuchs CS, Doi T, Jang RW, et al. Safety and efficacy of pembrolizumab monotherapy in patients with previously treated advanced gastric and gastroesophageal junction cancer: phase 2 clinical KEYNOTE-059 trial. JAMA Oncology 2018;4:e180013.

23. Huang J, Mo H, Zhang W, et al. Promising efficacy of SHR-1210, a novel anti-programmed cell death 1 antibody, in patients with advanced gastric and gastroesophageal junction cancer in China. Cancer 2019;125:742-9.

24. Motz GT, Coukos G. Deciphering and reversing tumor immune suppression. Immunity 2013;39:61-73.

25. Allen E, Jabouille A, Rivera LB, et al. Combined antiangiogenic and anti-PD-L1 therapy stimulates tumor immunity through HEV formation. Sci Transl Med 2017;9:eaak9679.

26. Lanitis E, Irving $M$. Targeting the tumor vasculature to enhance T cell activity. Curr Opin Immunol 2015;33:55-63.

27. Tang H, Wang Y, Chlewicki L, et al. Facilitating $T$ cell infiltration in tumor microenvironment overcomes resistance to PD-L1 blockade. Cancer Cell 2016;29:285-96.

28. Jain RK. Normalizing tumor vasculature with antiangiogenic therapy: a new paradigm for combination therapy. Nature Med 2001;7:987-9.

29. Huang Y, Yuan J, Righi E, et al. Vascular normalizing doses of antiangiogenic treatment reprogram the immunosuppressive tumor microenvironment and enhance immunotherapy. Proc Natl Acad Sci U S A 2012;109:17561-6.

30. Rakhra K, Bachireddy P, Zabuawala T, et al. CD4(+) $T$ cells contribute to the remodeling of the microenvironment required for sustained tumor regression 
upon oncogene inactivation. Cancer Cell 2010;18:485-98.

31. Zhao S, Ren S, Jiang T, et al. Low-dose apatinib optimizes tumor microenvironment and potentiates antitumor effect of PD-1/PD-L1 blockade in lung cancer. Cancer Immunol Res 2019;7:630-43.

Cite this article as: Wei Q, Yuan X, Li J, Xu Q, Ying J. PD-1 inhibitor combined with apatinib for advanced gastric or esophagogastric junction cancer: a retrospective study. Transl Cancer Res 2020;9(9):5315-5322. doi: 10.21037/tcr-20-1333
32. Osorio JC, Ni A, Chaft JE, et al. Antibody-mediated thyroid dysfunction during T-cell checkpoint blockade in patients with non-small-cell lung cancer. Ann Oncol 2017;28:583-9. 